\title{
A Comparison of Unsedated Colonoscopy and Flexible Sigmoidoscopy in the Family Medicine Setting: An LA Net Study
}

\author{
Lyndee Knox, PhD, Ricardo G. Hahn, MD, and Christianne Lane, MS
}

Background: Colonoscopy visualizes more of the colon than flexible sigmoidoscopy. This study compares the outcomes of an unsedated modified colon endoscopy (MCE) with flexible sigmoidoscopy (FS) in family medicine practice.

Methods: We conducted a retrospective chart review of existing clinical data to compare outcomes for 48 patients undergoing MCE and 35 patients undergoing FS at 3 family medicine practices in Los Angeles. Outcomes of interest included completion rates, number of complications, depth reached, anatomic site visualized, and information about the number and nature of clinical findings.

Results: No significant differences were found between MCE and FS regarding completion rates (83.3\% vs 75\%, respectively). Expected statistically significant differences were found between the 2 procedures in the anatomic site visualized $(P<.01)$ and depth reached $(P<.01)$. Clinical pathologies were identified in $58 \%$ of MCE patients and $37 \%$ of FS patients. Four adenocarcinomas were identified in the MCE group in the proximal region of the colon that could not have been detected by FS.

Conclusions: Findings from this study suggest that MCE can be an acceptable alternative to FS in office settings for colorectal cancer screening. (J Am Board Fam Med 2007;20:444-450.)

Family physicians routinely provide endoscopic screening services to their patients in the form of flexible sigmoidoscopy (FS). More than a decade ago, Selby et al reported a $60 \%$ reduction in colorectal cancer mortality among people undergoing screening sigmoidoscopy. ${ }^{1}$ However, traditional FS only reaches a depth of $60 \mathrm{~cm}$ and so excludes 80 to $100 \mathrm{~cm}$ of colon from examination. Recent studies have suggested that FS may miss as many as half the lesions in the colon, ${ }^{2,3}$ a problem that may be particularly pronounced among women. In a recent study comparing the detection of polyps by colonoscopy and sigmoidoscopy, FS identified only $35.2 \%$ of women with advanced colorectal neoplasia compared with $66.3 \%$ of matched men. ${ }^{4}$ In

This article was externally peer reviewed.

Submitted 6 October 2006; revised 23 March 2007; accepted 2 April 2007.

From the Department of Family Medicine (LK, RGH) and the Department of Preventative Medicine, Biostatistics (CL), University of Southern California, Los Angeles.

Funding: none.

Prior presentation: Unsedated colonoscopy in primary care practice. North American Primary Care Research Group. October 2005. Quebec City, Quebec, Canada.

Conflict of interest: none declared.

Corresponding author: Lyndee Knox, PhD, University of Southern California, Department of Family Medicine, 1420 San Pablo St., PMB-B205, Los Angeles, CA 90033 (E-mail: knox@usc.edu). contrast, standard colonoscopy allows $100 \%$ of the cecum (total colon) to be viewed in approximately $76 \%$ or greater of procedures ${ }^{3,5,6}$ and has been shown to be more sensitive than FS for detecting large adenomas and cancers. ${ }^{2,4,7}$

Although the US Preventative Services Task Force does not yet recommend the use of one particular method of colorectal cancer screening over another, it strongly recommends that clinicians screen adults with average risk for colorectal cancer with one of a variety of different screening methods, including colonoscopies, beginning at age 50 and then again every 10 years. ${ }^{8}$ The American Cancer Society makes similar recommendations for adults at average risk. ${ }^{9}$ An excellent overview of colorectal cancer screening recommendations and surrounding controversies is available in Ransohoff's 2005 review of the topic. ${ }^{10}$

Many patients, particularly those who are uninsured or underinsured, do not have access to colonoscopy as a screening option because of the few trained colonoscopists working in medically underserved areas. ${ }^{1-13}$ In Los Angeles County alone, community physicians report that their uninsured and publicly insured patients with indications can wait as long as 8 months for a colonoscopy, and that screening colonoscopies are simply 
unavailable (phone conversation with G. Floutsis, MD, Medical Director Clinica Msr. Oscar A. Romero Community Health Center, November 2005; e-mail communication with RD Yang, MD, PhD, Division of Gastroenterology and Liver Diseases, Keck School of Medicine, University of Southern California, March 2007).

One solution to the limited capacity for screening colonoscopies in the health care system is to train primary care physicians to perform colonoscopies in the primary care settings. Numerous previous studies have shown that, after the completion of appropriate training, family physicians can perform colonoscopies competently and safely in inpatient and outpatient settings with high patient satisfaction, few to no complications, and reliable and valid clinical findings. ${ }^{14-17}$ Unfortunately, licensing regulations in some states relating to the use of conscious sedation (required for colonoscopy) can make it cost prohibitive for family physicians and other primary care physicians to offer colonoscopy in their practices. In California, full conscious sedation must be administered in a facility that is fully licensed either by the Department of Health Services, the Joint Accreditation Commission of Hospitals and Health Organizations or the American Association of Ambulatory Health Centers (California Senate Bill 595 to 19990816 Amended).

Several studies have compared unsedated colonoscopy with sedated procedures and with FS in specialist settings and have found the unsedated procedure to be comparable to sedated colonscopy and FS in terms of patient tolerance, complications, and completion rates. In one of the earliest of these studies, Thiis-Evensen et al (2000) of Norway evaluated the efficacy of colonoscopy without sedation during screening examination in 451 adult patients. ${ }^{18}$ Completion rates and complication rates for unsedated and sedated colonoscopy with an adult endoscope were comparable. Currently, the procedure is the de facto standard of care for colorectal cancer screening by colonoscopy in small provincial clinics and hospitals in Norway (e-mail communication with E. Thiis-Evensen, MD, Department of Medicine Telemark Central Hispital, Skien; Department A of Medicine, Rikshospitalet University Hospital, Oslo, Norway, December 2003). In a gastroenterology setting, Wu et al (2003) obtained similar findings in a comparison of unsedated colonoscopy with an adult colonoscope and FS and using nursing staff to deliver the procedure. ${ }^{19}$ Thompson, Springer, and Anderson found no significant differences in patient tolerance and examination duration when comparing unsedated colonoscopy with a pediatric colonoscope and $\mathrm{FS}^{20}$

Studies comparing pediatric and adult colonoscopes have found few significant differences between the two in time to cecum, patient tolerance, and endoscopist perception of difficulty, ${ }^{21}$ but found a slight superiority in completion rates for the pediatric colonoscope. Saifudden et al (2000) reported higher completion rates in procedures using the pediatric colonoscope compared with those using adult colonoscopies, especially in women. ${ }^{22}$ Okamoto et al (2005) found better completion rates with the pediatric compared with adult colonoscope in patients with fixed, angulated colons. ${ }^{23}$

In 2002, in response to their uninsured and publicly insured patients' lack of access to screening colonoscopies, 4 clinicians from 3 family medicine practices involved with LA Net, a primary care practice-based research network, began offering unsedated colonoscopy with a pediatric endoscope to adult patients under guidelines recommended by the US Preventative Services Task Force and American Cancer Society and those outlined in Table 1. The clinicians opted to use a pediatric colonoscope in the procedure based on evidence demonstrating the basic comparability of the 2 devices and a slightly higher completion rate for procedures conducted using the pediatric endoscope. At each practice, modified colon endoscopy (MCE) was offered to all average-risk adult patients eligible for colorectal cancer screening as an alternative to both already-available on-site FS and referral to an off-site specialty clinic for sedated colonoscopy. In a few rare instances, MCE was offered to patients in higher-risk categories after they were referred for off-site sedated colonoscopy while they were waiting for their appointment. In these instances, the patients were likely to experience very lengthy wait times for an off-site appointment because of their insurance status.

All of the family physicians in this study acquired their skills for FS while in residency training. Three of the 4 acquired their skills in colonoscopy over 10 years of practice and continuing medical education procedural courses through the American Academy of Family Physicians and others. One clinician had received formal colonoscopy training 
Table 1. Guidelines for Modified Colon Endoscopy

Primary indications:

- Screening for colorectal cancer in asymptomatic patients according to ACS guidelines

- Rescreening of patients with history of treated colon cancer

-Known familial colon cancer history

- Diagnostic examinations:

-Evaluation of positive FOBT

-Evaluation of rectal bleeding

-Evaluation of change in bowel habits

-Follow-up of selected patients previously found to have benign lesions

Contraindications:

- Multiple previous abdominal surgical procedures

- Known active inflammatory bowel disease or diverticulitis

- Inability to complete or tolerate bowel prep

- Inability to cooperate because of mental illness, dementia or disability

Reasons to terminate procedure and refer to consultant endoscopist:

- Inability to advance endoscope with reasonable effort and within reasonable time

- Excessive patient discomfort

- Extensive diverticulosis with inability to identify lumen

- Discovery of active inflammatory bowel disease or diverticulitis

- Discovery of multiple lesions that will require extensive polypectomy

- Discovery of obstructing lesion

- Discovery of obvious cancer

- Visual recognition of incompletely removed dysplastic lesion

Lesions to biopsy using biopsy forceps:

- All polyps

- Most abnormal mucosa

Lesions to NOT biopsy using biopsy forceps:

- Diverticuli (perforation risk)

- Flat lesions within diverticuli (perforation risk)

Lesions to remove with snare:

- Pedunculated polyps

Lesions to NOT remove with snare:

- Broad-based polyps (perforation risk)

- Lesions within diverticuli (perforation risk)

during his residency training program before joining the faculty practice. Each received training from the endoscope manufacturer in the use of the equipment and was instructed by the lead investigator (RGH), who has extensive experience in GI endoscopy. All reviewed Hoff's recommendations for conducting unsedated colonoscopy. ${ }^{24}$ All clinicians were credentialed by the University of Southern California Faculty Practice Credentialing Committee to perform these procedures.
Table 2. Sample Characteristics for Modified Colon Endoscopy and Flexible Sigmoidoscopy Groups

\begin{tabular}{lcc}
\hline Characteristic & MCE $\dagger(\mathrm{n}=48)$ & FS $\neq(\mathrm{n}=35)$ \\
\hline Age $^{*}$ & & \\
$<40$ years & $2(4.2)$ & $6(17.1)$ \\
$40-49$ & $6(12.5)$ & $8(22.9)$ \\
$50-59$ & $16(33.3)$ & $12(34.3)$ \\
$60-69$ & $17(35.4)$ & $6(17.1)$ \\
$70+$ & $6(12.5)$ & $2(5.7)$ \\
Female & $16(33.3)$ & $14(40.0)$ \\
Ethnicity & & \\
Caucasian & $22(45.8)$ & $24(68.6)$ \\
Black/African & $4(8.3)$ & $1(2.9)$ \\
American & $14(29.2)$ & $5(14.3)$ \\
Latino & $5(10.4)$ & $4(11.4)$ \\
Asian & $2(4.2)$ & $1(2.9)$ \\
Other & & \\
\hline
\end{tabular}

${ }^{*} P<.05$.

†Mean, 59.6, SD, 12.5; range, 31.2-86.2.

$\ddagger$ Mean, 51.3, SD, 13.4; range, 25.7-80.0.

All data shown as N (\%). MCE, modified colon endoscopy; FS, flexible sigmoidoscopy.

The goal of this study was to determine whether MCE and FS conducted in a family medicine practice are comparable in terms of completion rates and number of complications, and to determine whether MCE allows the family physician to visualize more of the colon than FS.

\section{Methods \\ Patients}

Billing records were used to identify all patients who underwent MCE or FS at the 3 family medicine practices between 2003 and 2005. A total of 48 patients underwent MCE and 35 patients underwent FS during this period. Table 2 provides patient demographics.

\section{Data}

Data were abstracted from existing medical records by the lead investigator (RGH) and a research assistant as part of a quality improvement effort.

\section{Modified Colon Endoscopy}

Patients who opted for MCE received instructions about preprocedure colon preparation using a standard protocol. They also received the proper bowel cleansing solutions and tablets and were provided with instructions regarding proper positioning, relaxation techniques based on recommendations 
made by Hoff, ${ }^{24}$ and personnel expected to be in attendance. Patients were also instructed to give feedback to the clinician and other members of the team about the level of comfort/discomfort and any other symptoms that might arise, such as nausea, dizziness, and the urge to evacuate gas.

The patient was positioned in the left lateral decubitus position and the endoscopist performed a rectal examination to ascertain the presence of internal/external hemorrhoids, prostate size in men, and any possible obstruction. Patients could observe the procedure on a video monitor and were instructed to give feedback to the endoscopist throughout the procedure about their level of discomfort using a 10-point pain index scale in which 1 equated to no discomfort and 10 equated to the worst pain the patient had ever experienced. Patients were instructed that they could stop the procedure at any point by saying "stop."

During the procedure, the endoscopist explained the technical and anatomic markers to the patient. During procedures in which technical difficulties were encountered (including severe bowel spasms, persistent looping, obstruction, and poor visualization of the lumen), the endoscopist automatically terminated the procedure. During cases in which biopsy, ablation, or fulguration were indicated, the endoscopist explained these procedures to the patient and what he or she could expect to see on the video monitor.

A video processor (model EPX-2200, Fujinon, Wayne, NJ) with a pediatric $170-\mathrm{cm}$ endoscope (EC 250LP5, Fujinon) was used, and standard snare, biopsy forceps, and hot wire snares using an Earht Radiation Budget Experiment electrical power unit were also available. Once past the splenic flexure, a stiffening device, 1.4 or $1.6 \mathrm{~mm}$ in diameter (model 14700, Zutron Medical, Kansas City, MO), was introduced to avoid excessive looping. The clinician then advanced the scope further to examine the rest of the transverse and ascending colon. Appropriate biopsies and samplings were obtained. All suspicious lesions were biopsied and sent to pathology. Any excessive bleeding sites were electrocauterized. Finally, a reverse look at the rectum and anal verge was performed. After the procedure, the patient was instructed to rest for a few minutes and was allowed to use the bathroom if needed. The endoscopist discussed findings with the patient. The patient was allowed to go home if his or her vital signs were stable.

\section{Flexible Sigmoidoscopy}

Patients who opted for FS received instruction at the time of scheduling for the procedure and were provided with a Fleet enema to be administered 2 hours before arrival for the procedure. The patient was instructed at the time of arrival regarding positioning, members of the team expected to be present, and discomfort. The patient administered another Fleet enema in the office before the procedure. The patient was positioned in the standard left lateral decubitus position and a digital rectal examination was performed to look for any masses and to examine the prostate (in men). The endoscopist then performed a standard sigmoidoscopy using a Fujinon video endoscope with a standard sigmoidoscope. Appropriate biopsy of lesions and electrofulguration of any unusual bleeding sites were performed. A reverse look at the rectum and anal verge occurred. At the end of the procedure, findings were discussed with the patient, who was then discharged if their vital signs were stable.

\section{Outcome Variables}

To evaluate the viability of MCE as an alternative to FS, the procedures were compared across completion rates, complication rates, depth reached, anatomic site visualized, and clinical findings. For purposes of this study, completion of MCE was defined as visualization beyond the hepatic flexure and down the ascending colon with insertion beyond $110 \mathrm{~cm}$, unless there was a foreshortened colon because of colon resection. Completion of FS was defined as reaching the splenic flexure or insertion of the scope to a depth of $60 \mathrm{~cm}$. Site of maximum insertion was determined by direct visualization and identification of an anatomic site, which was correlated with the centimeter marks on the endoscope. Anatomic site visualization and identification were based on landmarks characteristic of the different parts of the colon. Although the cecum was not always visualized during MCE, completion of the study required visualization of the ascending colon with scope insertion at or beyond the 110-cm mark. During situations in which coiling was suspected, insertion of the stiffening wire resulted in the straightening of the scope and further visualization of the colon. Clinical findings recorded included those noted in any standard colonoscopy textbook and included hemorrhoids, fissures, polyps, and masses. The presence or ab- 
sence of carcinoma was confirmed through the pathology report.

\section{Analysis}

Patient demographics for the 2 groups were analyzed for equivalence using $t$ test (age) and Pearson's $\chi^{2}$ (gender and ethnicity). Completion data, complications, and clinical findings were compared using Pearson's $\chi^{2}$. Significant group differences in demographics were controlled for in comparison of clinical findings. SPSS software version 13 (SPSS Inc, Chicago, IL) was used for all analyses.

\section{Results}

A total of 83 patients underwent MCE $(n=48)$ or FS $(n=35)$ between 2003 and 2005 at the 3 practice sites. A significant difference was found between the 2 groups for age $\left(\mathrm{t}_{(79)}=2.83 ; P=\right.$ .006): patients in the MCE group were older than those in the FS group. The reasons for this difference are not clear but one might speculate that patients in the older age group, specifically those in their 60s and 70s, who previously may have been referred to a gastrointestinal laboratory may have been more likely to opt for the on-site MCE procedure than their younger counterparts. Self-perceived risk may also have influenced patients to opt for the MCE, with older patients being more likely to perceive greater risk than younger patients and therefore more likely to chose MCE. No statistically significant differences were found between the groups for gender or ethnicity. All further analyses controlled for age.

\section{Completion Rates}

The completion rate for MCE at the 3 practice sites was $83.3 \%$, which is not significantly different from the completion rate $(75 \%)$ obtained for FS. The main reason cited for failure to complete both procedures was patient discomfort: $8.3 \%$ of MCE patients and $11.4 \%$ of FS patients did not complete the procedure because of discomfort. In MCE, poor preparation was also cited as a reason for noncompletion in 3 cases $(6.3 \%)$.

\section{Depth Reached and Site Visualized}

The cecum was visualized but not intubated in $72.9 \%$ of the MCE patients. In $6.3 \%$ of MCE procedures there was a successful cecal intubation. Because of the limited length of FS equipment,
Table 3. Completion Rate, Site Visualized, Depth Reached, Complications

\begin{tabular}{|c|c|c|}
\hline & $\operatorname{MCE}(\mathrm{n}=48)$ & $\mathrm{FS}(\mathrm{n}=35)$ \\
\hline Procedure completed & $40(83.3)$ & $24(75.0)$ \\
\hline \multicolumn{3}{|l|}{ Reasons for noncompletion } \\
\hline Bleeding & $0(0.0)$ & $0(0.0)$ \\
\hline Discomfort & $4(8.3)$ & $4(11.4)$ \\
\hline Excessive looping & $0(0.0)$ & $0(0.0)$ \\
\hline Poor preparation & $3(6.3)$ & $0(0.0)$ \\
\hline Obstructing mass & $2(4.2)$ & $0(0.0)$ \\
\hline Not specified & $0(0.0)$ & $7(63.6)$ \\
\hline $\begin{array}{l}\text { Reached maximum depth* } \\
\text { (160 cm for ME/60 } \\
\mathrm{cm} \text { for FS) }\end{array}$ & $9(18.8)$ & $14(40.0)$ \\
\hline \multicolumn{3}{|l|}{ Anatomical site visualized* } \\
\hline Sigmoid colon & $0(0.0)$ & $1(2.9)$ \\
\hline Descending colon & $0(0.0)$ & $11(31.4)$ \\
\hline Splenic flexure & $2(4.2)$ & $12(34.3)$ \\
\hline Transverse colon & $6(12.5)$ & $9(25.7)$ \\
\hline Hepatic flexure & $1(2.1)$ & $0(0.0)$ \\
\hline Ascending colon & $35(72.9)$ & $0(0.0)$ \\
\hline Cecum & $3(6.3)$ & $0(0.0)$ \\
\hline \multicolumn{3}{|l|}{$\begin{array}{l}\text { Maximum depth }(\mathrm{cm}) \text { for } \\
\text { procedure* } †\end{array}$} \\
\hline $0-20$ & $0(0.0)$ & $1(2.9)$ \\
\hline $21-40$ & $0(0.0)$ & $6(17.1)$ \\
\hline $41-60$ & $3(6.3)$ & $28(80.0)$ \\
\hline $61-80$ & $1(2.1)$ & $0(0.0)$ \\
\hline $81-100$ & $3(6.3)$ & $0(0.0)$ \\
\hline $101-120$ & $7(14.6)$ & $0(0.0)$ \\
\hline $121-140$ & $15(31.3)$ & $0(0.0)$ \\
\hline $141-160$ & $19(39.6)$ & $0(0.0)$ \\
\hline $\begin{array}{l}\text { Complications reported } \\
\text { (bleeding, infection, } \\
\text { perforation, and other) }\end{array}$ & $0(0.0)$ & $0(0.0)$ \\
\hline
\end{tabular}

${ }^{*} P<.05$

†For MCE: mean, 130.1; SE, 30.1. For FS: mean, 50.6; SD, 10.0 .

All data presented as N (\%). MCE, modified colon endoscopy; FS, flexible sigmoidoscopy.

none of these sites could be visualized. Thus, as expected, statistically significant differences were found between the 2 procedures in anatomic site visualized $(P<.01)$. Similarly, when analyzed by depth readings on the endoscopes, MCE reached significantly further into the colon (mean, 130.1 $\mathrm{cm}$; SD, $30.1 \mathrm{~cm}$ ) than the FS (mean, $50.6 \mathrm{~cm}$; SD, $10.0 \mathrm{~cm})$; again showing an expected and statistically significant difference $(P<.01)$. A summary of findings is provided in Table 3.

\section{Complications}

No complications were reported in either group. 
Table 4. Descriptive Data About Clinical Findings for Modified Colon Endoscopy and Flexible Sigmoidoscopy

\begin{tabular}{lcc}
\hline & MCE (n=48) & FS (n=35) \\
\hline Normal Colon & $16(33.3)$ & $20(57.1)$ \\
Diverticula & $16(33.3)$ & $6(17.1)$ \\
Hemorrhoids & $4(8.3)$ & $4(11.4)$ \\
Inflammatory Bowel Disease & $3(6.3)$ & $0(0.0)$ \\
Mass lesions & $1(2.1)$ & $0(0.0)$ \\
Polyps & $6(12.5)$ & $4(11.4)$ \\
Adenocarcinoma & $4(8.3)$ & $0(0.0)$ \\
Hyperperistalisis & $2(4.2)$ & $4(11.4)$ \\
Other & $7(14.6)$ & $0(0.0)$ \\
\hline
\end{tabular}

MCE, modified colon endoscopy; FS, flexible sigmoidoscopy.

\section{Clinical Findings}

Pathology was identified in 58\% of MCE patients compared with $37 \%$ of FS patients. Adenocarcinomas were identified in 4 MCE patients compared with none in the FS group, a clinically significant finding. One of these was a younger patient (age 31) with a family history of colon cancer who opted to undergo MCE while he was waiting for traditional sedated colonoscopy through the LA County system. The second was a 61-year-old patient with a similarly high-risk family history who also opted to undergo MCE concurrent to a referral to a gastroenterologist for screening. The other 2 were patients with unexpected findings of cancerous polyps in the transverse colon. Tests of significance were not conducted with these data because of the small sample size and the statistically significant difference in the average age of the 2 groups; instead, these data were treated as descriptive. Clinical findings for the 2 procedures are summarized in Table 4.

\section{Variations in Outcome by Clinician}

Among the 4 clinicians who performed MCE across the 3 practice sites, no differences were found on completion rates, reasons for noncompletion, clinical findings, or depth reached $(P=.08)$. Similarly, among the 6 clinicians who performed FS there were no statistically significant differences found regarding completion rates, reasons for noncompletion, or depth reached $(P=.29)$ (Table 5).

\section{Discussion and Conclusions}

MCE achieved completion rates comparable to FS, no complications, and it allowed family physicians to visualize significantly greater portions of the
Table 5. Variations in Outcomes by Clinician

\begin{tabular}{|c|c|c|c|c|}
\hline & \multicolumn{2}{|c|}{$\mathrm{MCE}^{*}$} & \multicolumn{2}{|c|}{ FS $†$} \\
\hline & $\chi_{(3)}^{2}$ & $P$ & $\chi_{(5)}^{2}$ & $P$ \\
\hline Procedure completed & 2.98 & 0.40 & 1.86 & 0.87 \\
\hline \multicolumn{5}{|l|}{ Noncompletion } \\
\hline Discomfort & 3.15 & 0.37 & 3.75 & 0.59 \\
\hline Poor Preparation & 2.42 & 0.49 & - & - \\
\hline \multicolumn{5}{|l|}{ Findings } \\
\hline Normal Colon & 3.40 & 0.33 & 6.98 & 0.22 \\
\hline Diverticula & 0.65 & 0.87 & 5.24 & 0.39 \\
\hline Hemorrhoids & 2.36 & 0.50 & 7.72 & 0.17 \\
\hline $\begin{array}{l}\text { Infl. Bowel } \\
\text { Disease }\end{array}$ & 2.86 & 0.41 & - & - \\
\hline Mass Lesions & 0.54 & 0.91 & - & - \\
\hline Polyps & 5.33 & 0.15 & 0.233 & 0.80 \\
\hline Hyperperistalisis & 1.27 & 0.74 & - & - \\
\hline Other & 4.97 & 0.17 & 10.78 & 0.06 \\
\hline
\end{tabular}

* Number of clinicians $=4$.

$\dagger$ Number of clinicians $=6$.

MCE, modified colon endoscopy; FS, flexible sigmoidoscopy.

colon than is possible with FS. Using MCE, the family physicians in this study were able to visualize the cecum $72.9 \%$ of the time and to intubate the cecum in $6.3 \%$ of the cases. Based on these data, we conclude that MCE can be an acceptable alternative to FS for colorectal cancer screening in family practice.

Although its use in family practice is promising, it is important to note that MCE also has significant limitations. The rate of intubating the cecum in MCE is only $6.3 \%$, which is significantly lower than that achieved with regular sedated colonoscopy. Thus, even though MCE improves the family physician's ability to visualize more of the colon, it cannot be viewed as a replacement for traditional sedated colonoscopy. All patients with higher-risk indications should be referred for traditional sedated colonoscopy for screening and, until these limitations are overcome, MCE should only be used for screening adults with average risk and not for diagnostic evaluations.

The ability to extend a standard office sigmoidoscopy to encompass a significantly larger segment of the colon has the potential to significantly enhance family physicians' ability to detect cancers or potentially cancerous lesions in their patients. In the past, family physicians who provided colonoscopy services in their office or in a gastrointestinal endoscopy laboratory had to commit large segments of their time, thus disrupting their practice 
pattern. In some states, the costs of providing office-based colonoscopy is prohibitive because of regulations governing the use of conscious sedation. MCE greatly reduces the time, effort, and staffing requirements for offering screening colonoscopies in family medicine practices, and it eliminates the need for conscious sedation. Findings from the current study are encouraging and suggest that MCE can be an acceptable alternative to FS in the family medicine practice, and a simpler and more cost-effective alternative to traditional sedated colonoscopy.

Limitations to this study include lack of randomization and small sample size. A larger, randomized controlled trial is needed to evaluate the reach, effectiveness, and feasibility of offering MCE in family medicine and its acceptability to patients relative to FS and other methods of colorectal cancer screening.

We gratefully acknowledge the assistance of Michael Fong, MD; Carmela Lomonaco, PHD; Prapti Upadhyay, MA; and Laura Myerchin, MA, for their help in conducting this study.

\section{References}

1. Selby JV, Friedman GD, Quesenberry CP, Weiss NS. A case-controlled study of screening sigmoidoscopy and mortality from colorectal cancer. N Engl J Med 1999;326:653-7.

2. Lieberman DA, Weiss DG, Bond JH, Ahnen DJ, Garewal H, Chejfec G. Use of colonoscopy to screen asymptomatic adults for colorectal cancer. Veterans Affairs Cooperative Study Group 380. N Engl J Med 2000;343:162-8.

3. Imperiale TF, Wagner DR, Lin CY, Larkin GN, Rogge JD, Ransohoff DF. Risk of advanced proximal neoplasms in asymptomatic adults according to the distal colorectal findings. N Engl J Med 2000;343: 169-74.

4. Schoenfeld P, Cash B, Flood A, et al. Colonoscopic screening of average-risk women for colorectal neoplasia. N Engl J Med 2005;352:2061-8.

5. Nelson DB, McQuaid KR, Bond JH, et al. Procedural success and complications of large-scale screening colonoscopy. Gastrointest Endosc 2002;55:307-14.

6. Bowles CJA, Leicester R, Romaya C, et al. A prospective study of colonoscopy practice in the UK today: are we adequately prepared for national colorectal cancer screening tomorrow? Gut 2004;53:277-83.

7. Walsh JM, Terdiman JP. Colorectal cancer screening: scientific review. JAMA 2003;289:1288-96.

8. U.S. Preventive Services Task Force. Screening for Colorectal Cancer: Recommendations and Rationale. July 2002. Rockville (MD): Agency for Healthcare Research and Quality. Available at http://www.ahrq.gov/clinic/ 3rduspstf/colorectal/colorr.htm. Accessed May 2006.

9. Byers T, Levin B, Rothenberger D, Dodd GD, Smith RA. American Cancer Society guidelines for screening and surveillance for early detection of colorectal polyps and cancer: update 1997. American Cancer Society Detection and Treatment Advisory Group on Colorectal Cancer. CA Cancer 7 Clin 1997; 47:154-60.

10. Ransohoff D. Colon cancer screening in 2005: status and challenges. Gastroenterology 2005;125:1685-95.

11. Wendt E. Screening for colorectal cancer. N Engl J Med 2001;345:1851; author reply, 1852.

12. Aldigé CR, Alberts DS, Berdebes J, et al. Confronting colorectal cancer: action steps for change. Alexandria (VA): Cancer Research Foundation of America; 2002.

13. Seeff LC, Nadel MR, Klabunde CN, et al. Patterns and predictors of colorectal cancer test use in the adult US population. Cancer 2004;100:2093-103.

14. Newman R, Nichols D, Cummings DM. Outpatient colonoscopy by rural family physicians. Ann Fam Med 2005;3:122-5.

15. Hopper W, Kyker KA, Rodney WM. Colonoscopy by a family physician: a 9-year experience of 1,048 procedures. J Fam Pract 1996;43:561-6.

16. Pierzchajlo RPJ, Ackerman RJ, Vogel RL. Colonoscopy performed by a family physician. A case series of 751 procedures. J Fam Pract 1997;44:473-80.

17. Rodney WM, Dabov G, Cronin C. Evolving colonoscopy skills in a rural family practice: the first 293 cases. Fam Pract Res J 1993;13:43-52.

18. Thiis-Evensen E, Hoff GS, Sauar J, Vatn MH. Patient tolerance of colonoscopy without sedation during screening examination for colorectal polyps. Gastrointest Endosc 2000;52:606-10.

19. Wu K, Titzer D, Soetikno R, Triadafilopoulos G. Use of a colonosope instead of a simoidoscope to screen asymptomatic adults for colorectal cancer. Gastrointest Endosc 2003;58:720-4.

20. Thompson RD, Springer EW, Anderson PB. Patient tolerance of unsedated colonoscopy vs. flexible sigmoidoscopy in colon cancer screening. Gastrointest Endosc 2000;51:4.

21. Shumaker DA, Zaman A, Katon RM. A randomized controlled trial in a training institution comparing a pediatric variable stiffness colonoscope, a pediatric colonoscope, and an adult colonoscope. Gastrointest Endosc 2002;55:172-9.

22. Saifuddin T, Trivedi M, King PD, Madsen R, Marshall JB. Usefulness of a pediatric colonoscope for colonoscopy in adults. Gastrointest Endosc 2000;51:314-7.

23. Okamoto M, Kawabe T, Kato J, Yamaji Y, Ikenoue T, Omata M. Ultrathin colonoscope with a diameter of $9.8 \mathrm{~mm}$ for total colonoscopy. J Clin Gastroenterol 2005;38:679-83.

24. Hoff G. Colonoscopy without sedation. Scand J Gastroenterol 2000;35:225-6. 